\title{
On a Generalization of Euler's $\phi$-function
}

\author{
Ryūki Matsuda*
}

Professor Tanaka extended Euler's $\phi$-function and abtained the formula to give the value of the function [2]. In this note, we extend these further to any algebraic number field.

Let $\mathcal{O}$ be the ring of algebraic integers of a (finite) algebraic number field. Let $S_{a, \mathrm{~m}}$ be the set of elements $x+m$ of $\mathfrak{O} / \mathfrak{m}$ such that

$$
(x, \mathrm{~m})=(x+\alpha, \mathrm{m})=1
$$

for fixed $\alpha \in \mathfrak{O}$ and an (integral) ideal $\mathrm{m}$ of $\mathfrak{S}$.

Definimion. We define the generalized Euler's $\phi$-function $\phi_{\alpha}(\mathrm{m})$ to be the number of elements of $S_{a, \mathrm{~m}}$.

LeMma 1. Let $\mathrm{m}, \mathfrak{m}^{\prime}$ be ideals of $\mathfrak{O}$ such that

$\left(\mathrm{m}, \mathrm{m}^{\prime}\right)=1$,

then we have

$\phi_{\alpha}\left(m m^{\prime}\right)=\phi_{\alpha}(m) \phi_{a}\left(\mathrm{~m}^{\prime}\right)$.

Proof. We have

$0, \mathrm{mut}^{\prime} \cong 0 / \mathrm{m} T \mathrm{O} / \mathrm{m}^{\prime}$

by the map

$$
x+m \mathrm{~m}^{\prime} \longleftrightarrow\left(x+\mathrm{m}, x+\mathrm{m}^{\prime}\right) .
$$

And the result follows from the fact that

$$
\left(x, \mathfrak{m m}^{\prime}\right)=\left(x+\alpha, \mathrm{mm}^{\prime}\right)=1
$$

if and only if

$$
(x, \mathfrak{m})=(x+\alpha, \mathfrak{m})=\left(x, \mathfrak{m}^{\prime}\right)=\left(x+\alpha, \mathfrak{m}^{\prime}\right)=1 .
$$

Now let $T_{a, m}$ be the set of elements $x+\mathrm{m}$ of $D / \mathrm{m}$ such that

$$
x^{2}+\alpha x \equiv 0(\mathrm{~m}) \text {. }
$$

We set $N_{a, m}$ to be the number of elements of $T_{a, m}$. 
LeMMa 2. For a prime ideal $\mathfrak{p}$ of $\mathfrak{O}$ and a natural number a, we have

$$
\phi_{\alpha}\left(p^{a}\right)=N(\mathfrak{p})^{a}-N(\mathfrak{p})^{a-1} N_{a, p},
$$

where $N($ ) denotes the norm.

Proof. Let

$$
T_{a, \mathfrak{p}}=\left\{x_{1}+\mathfrak{p}, \ldots, x_{k}+\mathfrak{p}\right\}, k=N_{a, \text { }}
$$

and

$$
\mathfrak{p} / \mathfrak{p}^{a}=\left\{y_{1}+\mathfrak{p}^{a}, \ldots, y_{c}+\mathfrak{p}^{a}\right\}, c=N(\mathfrak{p})^{a-1} .
$$

For any $x$ of $\mathcal{D}$,

$$
x+\mathfrak{p}^{a} \in S_{a, b}{ }^{a}
$$

if and only if

$$
x^{2}+\alpha x \equiv 0(p) \text {. }
$$

Hence the complement of $S_{a, p^{a}}$ in $\mathfrak{O} / \mathfrak{p}^{a}$ is just

$$
\left.x_{1}+y_{1}+\mathfrak{p}^{a}, \ldots, x_{i}+y_{j}+\mathfrak{p}^{a}, \ldots, x_{k}+y_{c}+p^{a}\right\} .
$$

Therefore it holds :

$$
\phi_{a}\left(\mathfrak{p}^{a}\right)=N\left(\mathfrak{p}^{a}\right)-k c=N(\mathfrak{p})^{a}-N(\mathfrak{p})^{a-1} N_{\alpha, \mathfrak{p}} .
$$

PROPOSITION.

$\phi_{a}(\mathrm{~m} \mathrm{l})=N(\mathrm{~m}) \Pi\left(1-\frac{N_{a, \mathfrak{b}}}{N(\mathfrak{p})}\right)$.

Proof. Let

$$
\mathrm{m}=I 7 p^{a} .
$$

We have, by lemmas 1 and 2 ,

$$
\begin{aligned}
\phi_{a}(\mathfrak{m} \mathfrak{)}) & =\phi_{a}\left(\Pi \mathfrak{p}^{a}\right)=\Pi \phi_{a}\left(\mathfrak{p}^{a}\right) \\
& =\Pi\left(N(\mathfrak{p})^{a}-N(\mathfrak{p})^{a-1} N_{a, \mathfrak{p}}\right) \\
& =\Pi N(\mathfrak{p})^{a} I I\left(1-\frac{N_{a, \mathfrak{p}}}{N(\mathfrak{p})}\right) \\
& =N(\mathfrak{m}) \prod_{\mathfrak{p} \mid \mathfrak{n}}\left(1-\frac{N_{a, \mathfrak{p}}}{N(\mathfrak{p})}\right) .
\end{aligned}
$$

Corollary. If $\alpha \equiv 0(\mathrm{~m})$,

$$
\phi_{a}(\mathrm{iI})=N(\mathrm{iII}) \prod_{\mathfrak{p}, m}\left(1-\frac{1}{N(\mathfrak{p})}\right) .
$$


Proof. Because $N_{a, p}=1$ in this case.

Remark. We can define also a generalized Euler's $\phi$-function $\phi_{\mathfrak{l}}(\mathfrak{m})$ for an ideal $m$ of $\mathcal{D}$ and for a residue class $\mathfrak{R} \bmod m$. And it is obvious that we get the similar results.

\section{References}

[1] T. Takagi, Theory of algebraic numbers, Iwanami (1948).

[2] K. Tanaka, A generalization of Euler's $\phi$-function, Res. Rep. Tokyo Electrical Engrg. College, 15 (1967), 24-29. 